\title{
Prognostic effect of preoperative serum albumin to globulin ratio in patients treated with cytoreductive nephrectomy for metastatic renal cell carcinoma
}

\author{
Ekaterina Laukhtina $^{1,2} \wedge$, Benjamin Pradere ${ }^{1,3}$, David D’Andrea ${ }^{1}$, Giuseppe Rosiello ${ }^{4,5}$, Stefano Luzzago ${ }^{4,6}$, \\ Angela Pecoraro ${ }^{4,7}$, Carlotta Palumbo ${ }^{4,8}$, Sophie Knipper ${ }^{4,9}$, Pierre I. Karakiewicz ${ }^{4}$, Vitaly Margulis ${ }^{10}$, \\ Fahad Quhal $^{1,11}$, Reza Sari Motlagh ${ }^{1}$, Hadi Mostafaei ${ }^{1,12}$, Keiichiro Mori ${ }^{1,13}$, Victor M. Schuettfort ${ }^{1,14}$, \\ Dmitry Enikeev ${ }^{2}$ Shahrokh F. Shariat ${ }^{1,2,10,15,16,17,18,19}$
}

${ }^{1}$ Department of Urology, Comprehensive Cancer Center, Medical University of Vienna, Vienna, Austria; ${ }^{2}$ Institute for Urology and Reproductive Health, Sechenov University, Moscow, Russia; ${ }^{3}$ Department of Urology, University Hospital of Tours, Tours, France; ${ }^{4}$ Cancer Prognostics and Health Outcomes Unit, Division of Urology, University of Montreal Health Center, Montreal, Canada; ${ }^{5}$ Department of Urology and Division of Experimental Oncology, Urological Research Institute (URI), IRCCS San Raffaele Scientific Institute, Milan, Italy; ${ }^{6}$ Department of Urology, European Institute of Oncology, IRCCS, Milan, Italy; ${ }^{7}$ Department of Urology, San Luigi Gonzaga Hospital, University of Turin, Turin, Italy; ${ }^{8}$ Urology Unit, Department of Medical and Surgical Specialties, ASST Spedali Civili of Brescia, Radiological Science and Public Health, University of Brescia, Brescia, Italy; ${ }^{9}$ Martini-Klinik Prostate Cancer Center, University Hospital Hamburg-Eppendorf, Hamburg, Germany; ${ }^{10}$ Department of Urology, University of Texas Southwestern Medical Center, Dallas, TX, USA; ${ }^{11}$ Department of Urology, King Fahad Specialist Hospital, Dammam, Saudi Arabia; ${ }^{12}$ Research Center for Evidence Based Medicine, Tabriz University of Medical Sciences, Tabriz, Iran; ${ }^{13}$ Department of Urology, The Jikei University School of Medicine, Tokyo, Japan; ${ }^{14}$ Department of Urology, University Medical Center Hamburg-Eppendorf, Hamburg, Germany; ${ }^{15}$ Department of Urology, Weill Cornell Medical College, New York, NY, USA; ${ }^{16}$ Department of Urology, Second Faculty of Medicine, Charles University, Prague, Czech Republic; ${ }^{17}$ Karl Landsteiner Institute of Urology and Andrology, Vienna, Austria; ${ }^{18}$ Division of Urology, Department of Special Surgery, Jordan University Hospital, The University of Jordan, Amman, Jordan; ${ }^{19}$ European Association of Urology Research Foundation, Arnhem, The Netherlands

Contributions: (I) Conception and design: SF Shariat, D Enikeev, PI Karakiewicz, V Margulis; (II) Administrative support: SF Shariat, V Margulis; (III) Provision of study materials or patients: SF Shariat, V Margulis; (IV) Collection and assembly of data: All authors; (V) Data analysis and interpretation: All authors; (VI) Manuscript writing: All authors; (VII) Final approval of manuscript: All authors.

Correspondence to: Shahrokh F. Shariat, MD. Department of Urology, Comprehensive Cancer Center, Vienna General Hospital, Medical University of Vienna, Währinger Gürtel 18-20, 1090, Vienna, Austria. Email: shahrokh.shariat@meduniwien.ac.at.

Background: Accurate identification of ideal candidates for cytoreductive nephrectomy (CN) for metastatic renal cell carcinoma (mRCC) is an unmet need. We tested the association between preoperative value of systemic albumin to globulin ratio (AGR) and overall survival (OS) as well as cancer-specific survival (CSS) in mRCC patients treated with $\mathrm{CN}$.

Methods: mRCC patients treated with CN were included. The overall population was therefore divided into two AGR groups using cut-off of 1.43 (low, <1.43 vs. high, $\geq 1.43$ ). Univariable and multivariable Cox regression analyses tested the association between AGR and OS as well as CSS. The discrimination of the model was evaluated with the Harrel's concordance index (C-index). The clinical value of the AGR was evaluated with decision curve analysis (DCA).

Results: Among 613 mRCC patients, 159 (26\%) patients had an AGR <1.43. Median follow-up was 31 (IQR: 16-58) months. On univariable analysis, low preoperative serum AGR was significantly associated with both OS (HR: 1.55, 95\% CI: 1.26-1.89, P<0.001) and CSS (HR: 1.55, 95\% CI: 1.27-1.90, P<0.001). On multivariable analysis, AGR $<1.43$ was associated with worse OS (HR: 1.51, 95\% CI: 1.23-1.85,

^ ORCID: 0000-0002-8953-0272. 
$\mathrm{P}<0.001$ ) and CSS (HR: 1.52, 95\% CI: 1.24-1.86, $\mathrm{P}<0.001)$. The addition of AGR only minimally improved the discrimination of a base model that included established clinicopathologic features (C-index=0.640 vs. $\mathrm{C}$-index=0.629). On DCA, the inclusion of AGR marginally improved the net benefit of the prognostic model. Low AGR remained independently associated with OS and CSS in the IMDC intermediate risk group (HR: 1.52, 95\% CI: 1.16-1.99, P=0.002).

Conclusions: In our study, low AGR before CN was associated with worse OS and CSS, particularly in intermediate risk patients.

Keywords: Metastatic renal cell carcinoma (mRCC); cytoreductive nephrectomy (CN); overall survival (OS); cancer-specific survival (CSS); albumin to globulin ratio (AGR)

Submitted Jul 17, 2020. Accepted for publication Dec 04, 2020.

doi: $10.21037 /$ tau-20-1101

View this article at: http://dx.doi.org/10.21037/tau-20-1101

\section{Introduction}

Approximately $25 \%$ of patients with newly diagnosed renal cell carcinoma (RCC) present with metastatic disease (mRCC) in industrialized countries (1). Therapeutic strategies for $\mathrm{mRCC}$ are based on the International metastatic renal cell carcinoma Database Consortium (IMDC) risk stratification (2). For IMDC favorable and intermediate risk patients cytoreductive nephrectomy $(\mathrm{CN})$ remains a valuable treatment option (3). Ideally, preoperative biomarkers could help identify the right therapy for the right patient. However, preoperative biomarkers able to predict either treatment response or other oncological outcomes in mRCC patients are still lacking. Inflammation and the immune response play a major role in tumor development, proliferation and metastatic spreading (4). Several markers of systemic inflammatory response have been already investigated in RCC (5-9). However, none of them have been yet implemented in the clinical decision making (3).

Albumin and globulins are two major serum proteins that play a pivotal role in the inflammatory process (10). Instead of the absolute concentration of albumin and globulin, albumin to globulin ratio (AGR) is a ratio that combines the two indexes. Previously, AGR was mainly used for the diagnosis of liver function and immunological diseases (11). Recently, AGR, which reflects the systemic inflammatory response and the systemic nutritional status, has shown prognostic ability in predicting oncological outcomes in various malignancies (12-15).

Previous studies have already suggested that AGR could be a potential biomarker to predict overall survival (OS), disease-free survival (DFS) and cancer-specific survival (CSS) in patients with localized or locally advanced RCC (16-18). Specifically, low preoperative AGR was associated with worse survival. However, the value of AGR as a predictor of oncological outcomes in patients treated with $\mathrm{CN}$ remains uninvestigated.

This study tested the association between preoperative serum AGR and oncological outcomes in mRCC patients treated with CN, after accounting for IMDC risk stratification. We present the following article in accordance with the STROBE reporting checklist (available at: http:// dx.doi.org/10.21037/tau-20-1101).

\section{Methods}

\section{Study design}

We retrospectively reviewed our established international multicenter database to identify mRCC patients treated with $\mathrm{CN}$ at tertiary centers in the United States and Europe. We excluded patients with other malignant primaries tumors mRCC. However, concomitant hematologic, liver diseases, chronic inflammatory disease including autoimmune disorder and infection within the last 12 months were not excluded.

The study was conducted in accordance with the Declaration of Helsinki (as revised in 2013). This study was reviewed and approved by the University of Texas Southwestern (Dallas, Texas, USA) Institutional Review Board (UT SW IRB File 0698 26900). Because of the retrospective nature of the research, the requirement for informed consent was waived. 


\section{Management and follow-up}

Dedicated uro-pathologists assigned pathologic stage according to the 2010 American Joint Committee on Cancer (AJCC) tumor, node and metastasis (TNM) staging system. All pathology reports from prior to 2010 were reviewed according to 2010 criteria. According to the IMDC patients were stratified into favorable $v s$. intermediate $v s$. poor risk groups (2).

All laboratory tests were done within one month prior to $\mathrm{CN}$. Serum AGR value has been calculated as baseline serum albumin to baseline total protein-baseline serum albumin ratio. To define the optimal pretreatment AGR cut-off value, we carried out a time-dependent receiver operating characteristic curve analysis for 3-year OS as the end-point, considering the median OS time (12 months). The median value of AGR was calculated as 1.59 (IQR: 1.39-1.78). The cut-off of 1.43 was determined as having the maximum Youden index value. The overall population was therefore divided into two AGR groups using this cutoff (lower $<1.43 v s$. higher $\geq 1.43$ ). OS time was calculated from the date of $\mathrm{CN}$ to death or last follow-up. CSS time was calculated from the date of $\mathrm{CN}$ to death from disease or last follow-up.

\section{Statistical analysis}

Associations between AGR status and categorical variables were assessed using $\chi^{2}$ or Fisher's exact tests. Differences in continuous variables were analyzed with the MannWhitney $U$ test. Univariable and multivariable Cox regression analyses tested the association of AGR with OS and CSS. The risk of survival was expressed as hazard ratios (HR) and 95\% CIs. Kaplan-Meier survival curves were used to graphically depict the association between AGR and survival. The log-rank test was used to determine the statistical difference between the AGR $<1.43$ and AGR $\geq 1.43$ groups with respect to death. The discrimination of the model was evaluated using the Harrel's concordance index (C-index). The clinical value of the AGR was evaluated with decision curve analysis (DCA) $(19,20)$. Statistical significance was set at $\mathrm{P}<0.05$. All tests were 2-sided. Analyses were performed using $\mathrm{R}$ version 3.6.2. (2009-2020 RStudio, Inc.).

\section{Results}

Overall, 613 patients were included in the analyses. Among them, 159 (26\%) patients had low AGR status (AGR <1.43). Patient characteristics are shown in Table 1. The number of patients with liver metastasis was significantly higher in patients with low preoperative AGR in contrast to those with high AGR (10.7\% vs. 5.3\%, $\mathrm{P}=0.03)$.

At median follow-up of 31 (IQR 16-58) months, a total of $472(77 \%)$ patients died and $99 \%$ of deaths were due to mRCC. Kaplan-Meier analyses showed a significant reduction in OS $(\log$-rank test $\mathrm{P}<0.001)$ and CSS $(\log$ rank test $\mathrm{P}<0.001)$ in patients with a preoperative low AGR (Figure 1). Median OS was 12 months (95\% CI: 1015 months) and 22 months (95\% CI: 19-25 months) in patients with low and high AGR, respectively.

On univariable analysis, low preoperative serum AGR was associated with lower OS (HR: 1.55, 95\% CI: 1.26-1.89, $\mathrm{P}<0.001)$ and lower CSS (HR: $1.55,95 \% \mathrm{CI}$ : $1.27-1.90, \mathrm{P}<0.001$ ) (Table 2). These results were confirmed in multivariable analyses, which adjusted for the effect of established clinicopathologic features. Low AGR levels remained significantly associated with worse OS (HR: 1.51, 95\% CI: $1.23-1.85, \mathrm{P}<0.001$ ) and worse CSS (HR: 1.52, 95\% CI: $1.24-1.86, \mathrm{P}<0.001)$. The addition of AGR improved the discrimination of a base model that included established clinicopathologic features $(\mathrm{C}$-index $=0.640 v s$. C-index $=0.629$ ). In DCA, the model including IMDC risk groups, type of systemic therapy, BMI and sarcomatoid features led to superior outcomes for any decision associated with a threshold probability abode $25 \%$. The inclusion of the AGR marginally improved the net benefit of the base model by a clinically non-significant margin (Figure 2).

In subgroup analyses of intermediate IMDC risk patients, preoperative serum AGR $<1.43$ was associated with significantly lower OS and CSS rates (HR: 1.52, 95\% CI: $1.17-1.98, \mathrm{P}=0.002)$. Similarly, in poor risk patients, low preoperative serum AGR was associated with worse OS and CSS rates (HR: 1.70, 95\% CI: 1.00-2.89, $\mathrm{P}=0.04$ ) (Figure 3). After adjusting for possible confounders on multivariable analyses, low AGR remained independently associated with worse OS and CSS in the intermediate risk group (both: HR: 1.52, 95\% CI: 1.16-1.99, $\mathrm{P}=0.002$ ) (Table 3) but not in the high risk group (all $\mathrm{P}>0.05$ ). No statistically significant association was recorded in favorable risk patients (all $\mathrm{P}>0.05$ ).

In the subgroup of 111 patients with sarcomatoid features, low preoperative AGR was associated with both OS and CSS (both: HR: 1.79, 95\% CI: 1.16-2.76, $\mathrm{P}=0.01$ ) in univariable analysis (Figure 4). After adjusting for possible 
Table 1 Clinicopathologic features of 613 patients treated with cytoreductive nephrectomy for metastatic renal cell carcinoma, stratified according to preoperative serum albumin to globulin ratio (AGR)

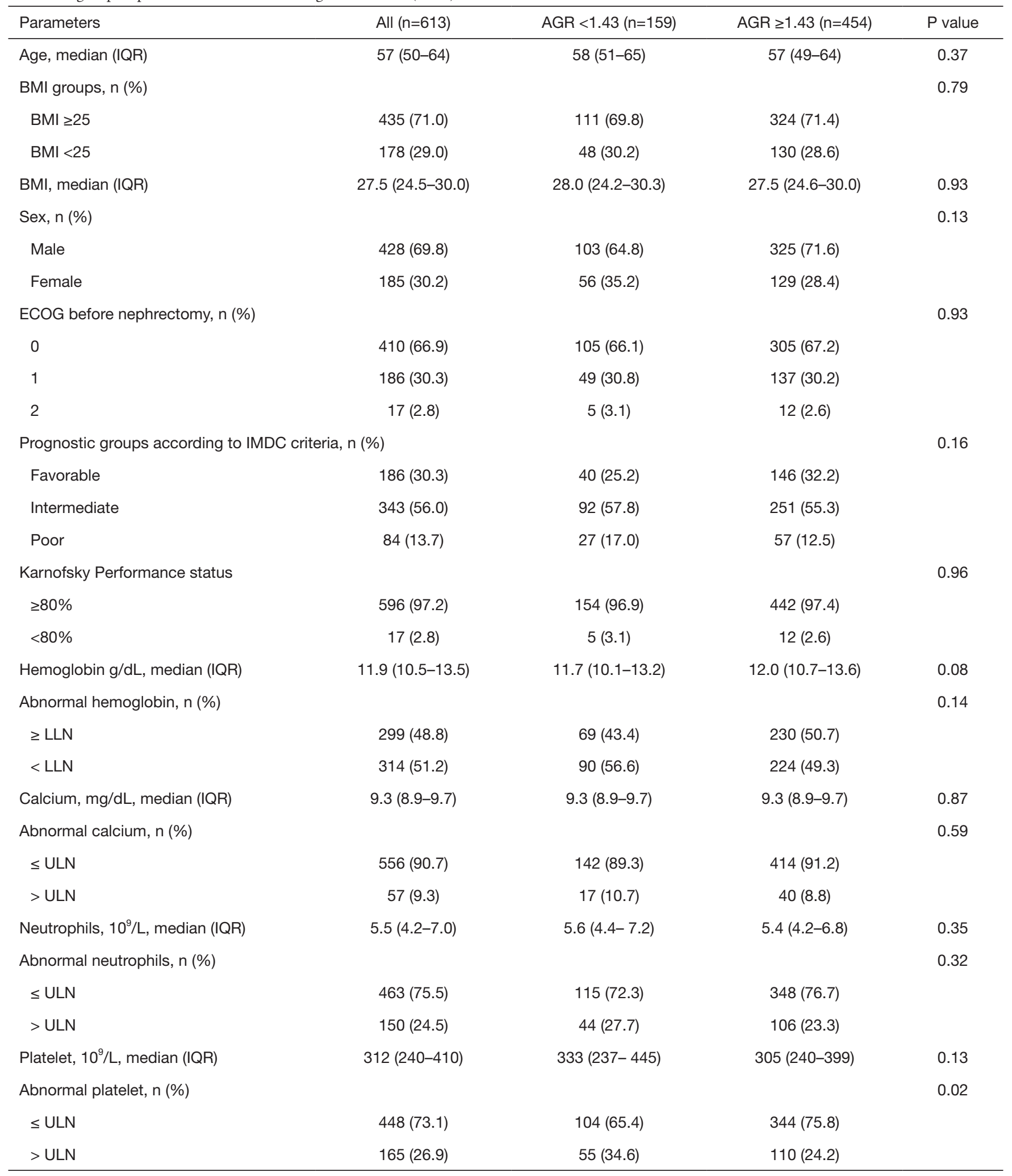

Table 1 (continued) 
Table 1 (continued)

\begin{tabular}{|c|c|c|c|c|}
\hline Parameters & All $(n=613)$ & AGR $<1.43(n=159)$ & AGR $\geq 1.43(n=454)$ & $P$ value \\
\hline Number of metastasis sites, $\mathrm{n}(\%)$ & & & & 0.3 \\
\hline Single & $370(60.4)$ & $90(56.6)$ & $280(61.7)$ & \\
\hline Multiple & $243(39.6)$ & $69(43.4)$ & $174(38.3)$ & \\
\hline Adrenal glands & $91(14.8)$ & $27(17.0)$ & $64(14.1)$ & 0.45 \\
\hline Bones & $181(29.5)$ & $43(27.0)$ & $138(30.4)$ & 0.49 \\
\hline Brain & $20(3.3)$ & $5(3.1)$ & $15(3.3)$ & 1 \\
\hline Liver & $41(6.7)$ & $17(10.7)$ & $24(5.3)$ & 0.03 \\
\hline Other & $38(6.2)$ & $6(3.8)$ & $32(7.0)$ & 0.2 \\
\hline Time from diagnosis to nephrectomy & & & & 0.2 \\
\hline Less than 12 months & $584(95.3)$ & $148(93.1)$ & $436(96.0)$ & \\
\hline More than 12 months & $29(4.7)$ & $11(6.9)$ & $18(4.0)$ & \\
\hline Lymphadenectomy, n (\%) & & & & 0.14 \\
\hline No & $361(58.9)$ & $102(64.2)$ & $259(57.0)$ & \\
\hline Yes & $252(41.1)$ & $57(35.8)$ & $195(43.0)$ & \\
\hline Histology, n (\%) & & & & 0.47 \\
\hline Grade, n (\%) & & & & 0.31 \\
\hline G1/G2 & $65(10.6)$ & $13(8.2)$ & $52(11.5)$ & \\
\hline G3/G4 & $548(89.4)$ & $146(91.8)$ & $402(88.5)$ & \\
\hline Neoadjuvant therapy, n (\%) & & & & 0.45 \\
\hline No & $500(81.6)$ & $126(79.2)$ & $374(82.4)$ & \\
\hline Yes & $113(18.4)$ & $33(20.8)$ & $80(17.6)$ & \\
\hline Adjuvant therapy, n (\%) & & & & 0.29 \\
\hline No & $132(21.5)$ & $29(18.2)$ & $103(22.7)$ & \\
\hline Yes & $481(78.5)$ & $130(81.8)$ & $351(77.3)$ & \\
\hline
\end{tabular}

IQR, interquartile range; BMI, body mass index; ECOG, Eastern Cooperative Oncology Group performance status; IMDC, International Metastatic RCC Database Consortium Risk Model; LLN, lower limit of normal; ULN, upper limit of normal. 

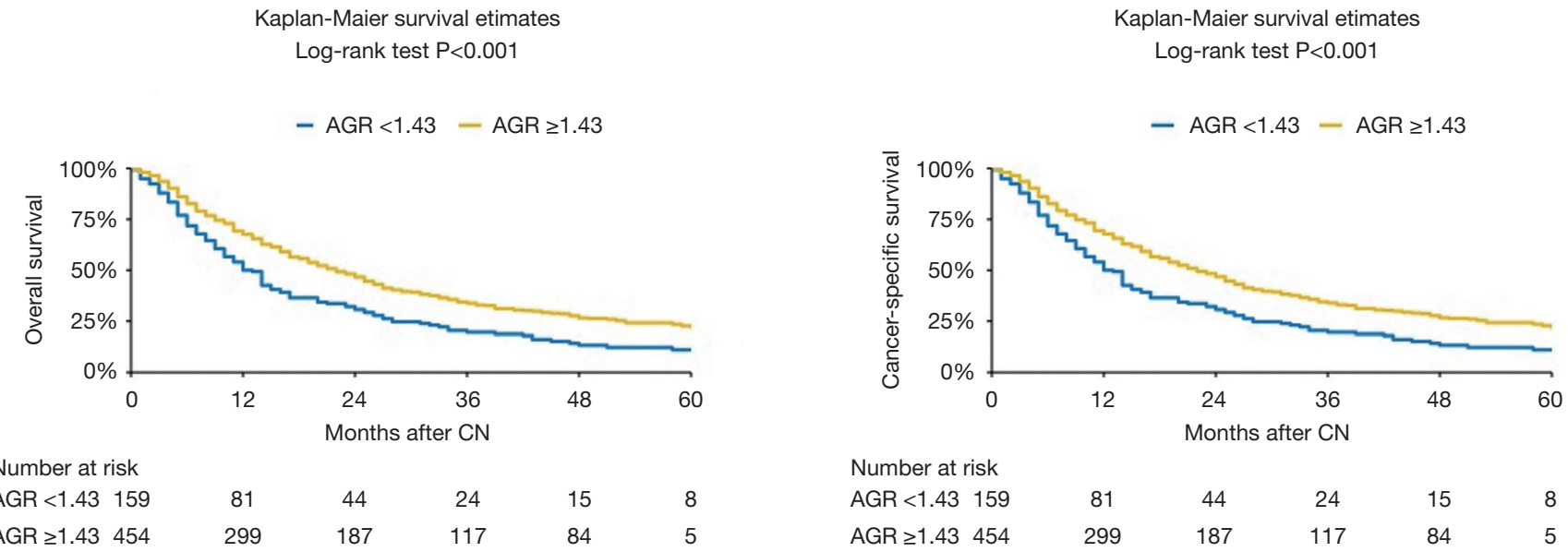

Figure 1 Kaplan-Meier analysis for overall and cancer-specific survival in 613 patients treated with cytoreductive nephrectomy (CN) for metastatic renal cell carcinoma, stratified according to preoperative serum albumin to globulin ratio (AGR) at a cut-off of 1.43 .

Table 2 Univariable and multivariable Cox regression analysis predicting OS and CSS in patients treated with cytoreductive nephrectomy for metastatic renal cell carcinoma

\begin{tabular}{|c|c|c|c|c|c|c|c|c|}
\hline \multirow{3}{*}{ Variables } & \multicolumn{4}{|c|}{ Os } & \multicolumn{4}{|c|}{ CSS } \\
\hline & \multicolumn{2}{|c|}{ Univariable } & \multicolumn{2}{|c|}{ Multivariable } & \multicolumn{2}{|c|}{ Univariable } & \multicolumn{2}{|c|}{ Multivariable } \\
\hline & $\mathrm{HR}(95 \% \mathrm{Cl})$ & $P$ value & $\mathrm{HR}(95 \% \mathrm{Cl})$ & $P$ value & $\mathrm{HR}(95 \% \mathrm{Cl})$ & $P$ value & $\mathrm{HR}(95 \% \mathrm{Cl})$ & $P$ value \\
\hline Age & 1 & 0.33 & 1.01 & 0.01 & 1 & 0.38 & 1.01 & 0.01 \\
\hline Sex (female) & 1.05 & 0.61 & 0.91 & 0.38 & 1.04 & 0.71 & 0.9 & 0.3 \\
\hline$E C O G \geq 1$ & 1.05 & 0.65 & 1.07 & 0.49 & 1.04 & 0.68 & 1.07 & 0.51 \\
\hline \multicolumn{9}{|l|}{ IMDC criteria } \\
\hline Favorable & Ref & Ref & Ref & Ref & Ref & Ref & Ref & Ref \\
\hline Intermediate & 1.19 & 0.09 & 1.05 & 0.63 & 1.2 & 0.08 & 1.06 & 0.57 \\
\hline Multiple metastatic sites & 1.43 & $<0.001$ & 1.51 & $<0.001$ & 1.44 & $<0.001$ & 1.51 & $<0.001$ \\
\hline Clear cell carcinoma histology & 0.55 & $<0.001$ & 0.54 & $<0.001$ & 0.55 & $<0.001$ & 0.54 & $<0.001$ \\
\hline Sarcomatoid features & 1.94 & $<0.001$ & 1.87 & $<0.001$ & 1.95 & $<0.001$ & 1.89 & $<0.001$ \\
\hline C-index with AGR & 0.64 & & & & 0.642 & & & \\
\hline C-index without AGR & 0.629 & & & & 0.63 & & & \\
\hline
\end{tabular}

OS, overall survival; CSS, cancer-specific survival; BMI, body mass index; ECOG, Eastern Cooperative Oncology Group performance status; IMDC, International Metastatic RCC Database Consortium Risk Model; AGR, albumin globulin ratio. 


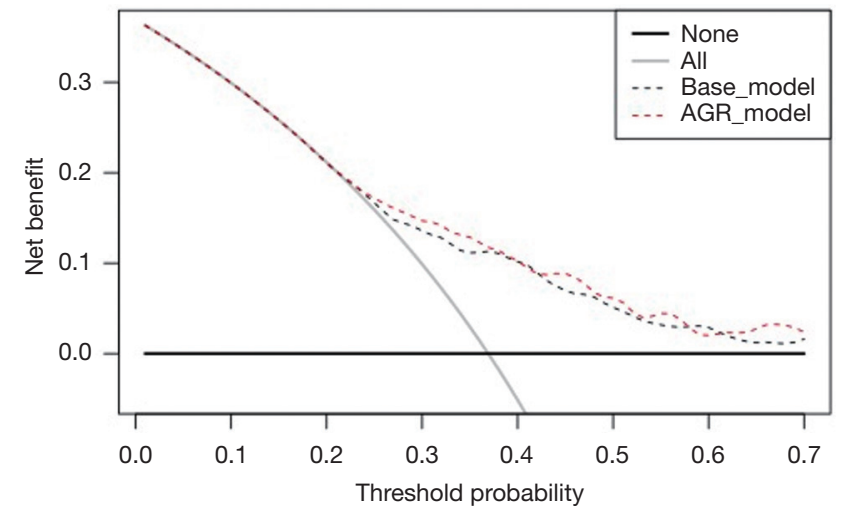

Figure 2 Decision curve analysis assessing the clinical impact of current prognostic models (Base model) with the integration of the albumin to globulin ratio (AGR model) estimating overall mortality at 1 year, in 613 patients treated with cytoreductive nephrectomy for metastatic renal cell carcinoma. The two models are compared with the strategies of treating all or none of the patients with cytoreductive nephrectomy.

confounders on multivariable analyses, AGR was not associated with neither OS nor CSS (all $\mathrm{P}>0.05$ ). In the subgroup of patients treated with tyrosine kinase inhibitor (TKI) therapy, AGR was neither associated with OS nor CSS (all $\mathrm{P}>0.05$ ).

\section{Discussion}

The exact mechanism underlying the association between low AGR and increased tumor aggressiveness is still unclear. It is hypothesized that this relationship results from a cascade of inflammatory mediators during the systematic inflammation that accompanies tumor progression (4). Both albumins and globulins are related to immunoinflammatory reactions and nutrition status in the human body (10). In presence of inflammation, inflammatory response cytokines, such as interleukin-1, interleukin-6 and TNF (tumor necrosis factor alpha), inhibit the synthesis of albumin (21). Furthermore, chronic inflammation results in vascular endothelial damages and increases vascular permeability (22). As a result, albumin permeates the interstitial space and its serum level decreases (23). This would suggest that low AGR (low albumin-high globulin), results from the inflammatory microenvironment induced by cancer cells, could predict poor oncologic prognosis.

In the present study, we investigated the association of preoperative serum AGR with oncologic outcomes in patients with mRCC and found an association with both OS and CSS. Moreover, after adjusting for the effects of established clinicopathologic features, AGR retained its statistical significance. These findings are in agreement with a previous retrospective analysis of 592 patients treated with radical or partial nephrectomy for localized or locally advanced RCC, which reported that low AGR level with a cut-off of 1.22 was associated with worse OS (HR: 6.529, 95\% CI: 3.036-14.042, $\mathrm{P}<0.001$ ) and worse CSS (HR: 8.806, 95\% CI: 3.891-19.928, $\mathrm{P}<0.001)(16)$. The inclusion of AGR in a prognostic model had a better prediction accuracy of 3 - and 5 -year OS than without the AGR $(\mathrm{C}$-index $=0.884 v s$. C-index $=0.806)$. In our study, the addition of AGR only marginally improved the discrimination of a base model (C-index $=0.640 v s$. C-index $=0.629)$. Two other studies reported similar results. In 162 patients with localized and locally advanced RCC, OS and DFS were found to be significantly lower in patients with an $\mathrm{AGR}<1.40$ group $(\mathrm{P}=0.006$ and $\mathrm{P}=0.012)$ (18). A study of 895 patients with RCC from all stages, with an AGR cut-off value of 1.47 , found an independent association of low AGR with OS (HR: 0.63, 95\% CI: 0.430.93, $\mathrm{P}=0.022$ ) (17). However, conventional multivariable analyses are not sufficient to demonstrate that a biomarker has a clinical benefit (19). Indeed, to explore this, in contrast to the above-mentioned studies, we used DCA (24). Our study showed that preoperative serum AGR marginally improved the net benefit of the base model by a clinically non-significant margin. Many explanations for the discrepancy of our results with that of others are possible. First, our population included only patients with metastatic disease, which is very different from locally and locally advanced diseases in terms of immunomodulation and systemic inflammation. Second, systemic treatment with TKI was used according to historic recommendations prior to current treatment strategies. Ideally, contemporary predictive value of AGR should be assessed in the era of immune checkpoint inhibitors (25). Due to its strong association with immuno-inflammatory reactions, AGR might represent a valuable prognostic and predictive marker for mRCC patients treated with immune checkpoint inhibitors as it has been already shown in non-small cell lung cancer (26).

It is also important to consider that combining AGR with other biomarkers may improve the accuracy of prognosis models. Several other prognostic serum-based biomarkers have been investigated in RCC patients $(7,27)$. For instance, 

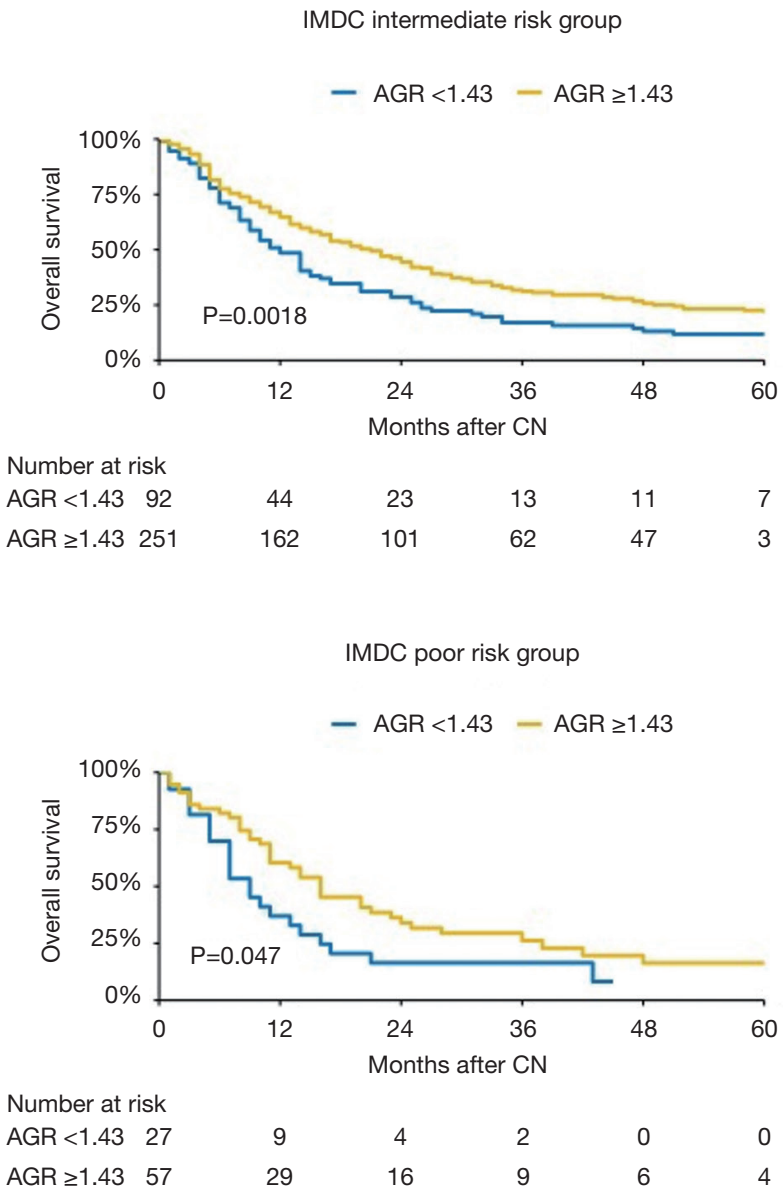
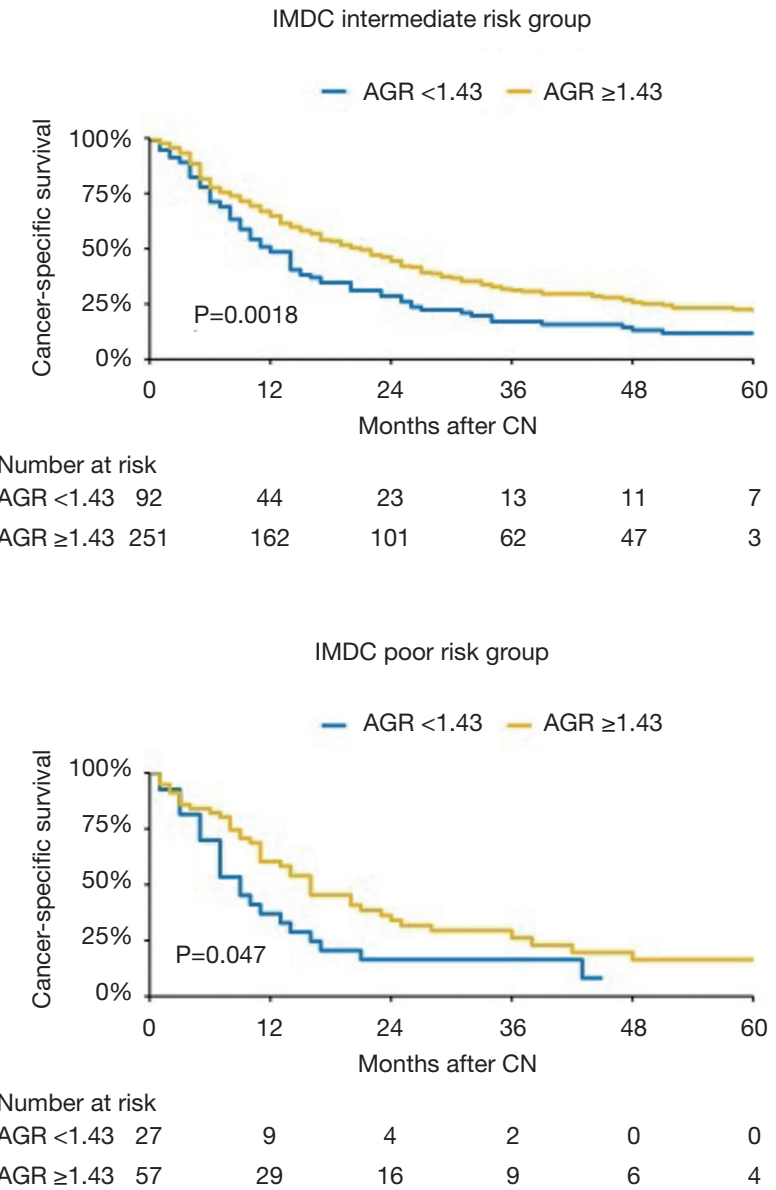

Figure 3 Overall and cancer-specific survival in patients treated with cytoreductive nephrectomy (CN) for intermediate risk metastatic renal cell carcinoma, according to the International Metastatic renal cell carcinoma Database Consortium (IMDC) prognostic model and stratified according to preoperative serum albumin to globulin ratio (AGR) at a cut-off of 1.43 .

neutrophil to lymphocyte ratio and C-reactive protein were associated with OS in mRCC (9). Other markers of systemic inflammation, lymphocyte to monocyte ratio and systemic inflammation response index, were associated with OS and pathological parameters in mRCC patients (28). Increased pretreatment platelet to lymphocyte ratio was an independent prognostic marker in patients treated with TKI for mRCC (6). In our study, AGR was not shown to be associated with OS or CSS in mRCC patients treated with TKI therapy. Most of the aforementioned studies were limited by their retrospective designs (29). Moreover, among the weaknesses of these studies, biomarker models were not compared with the well-established IMDC model. Further intensive studies to identify optimal predictive and prognostic biomarkers in a panel of biomarkers for the heterogenous malignancy that is $\mathrm{mRCC}$ are needed.

Our study is not devoid of limitations. The main limitation of the study was its retrospective and multicenter design, which may result in selective bias, lack of standardized laboratory, pathological, surgical and treatment approaches that could confound the results. The systemic therapy was heterogenous regarding drugs and their mechanisms of actions. Another limitation of our study is the fact that AGR might have been biased by the presence of an undetected liver, hematologic or immunologic diseases or a drug interaction that may have affected a blood protein levels of albumin and globulins. Additionally, AGR was assessed preoperatively at a single time point. AGR variability over time, in response to therapy and its relationship to the oncological prognosis of 
Table 3 Multivariable Cox regression analyses predicting OS and CSS in patients treated with cytoreductive nephrectomy for intermediate and poor risk metastatic renal cell carcinoma, according to the IMDC prognostic model

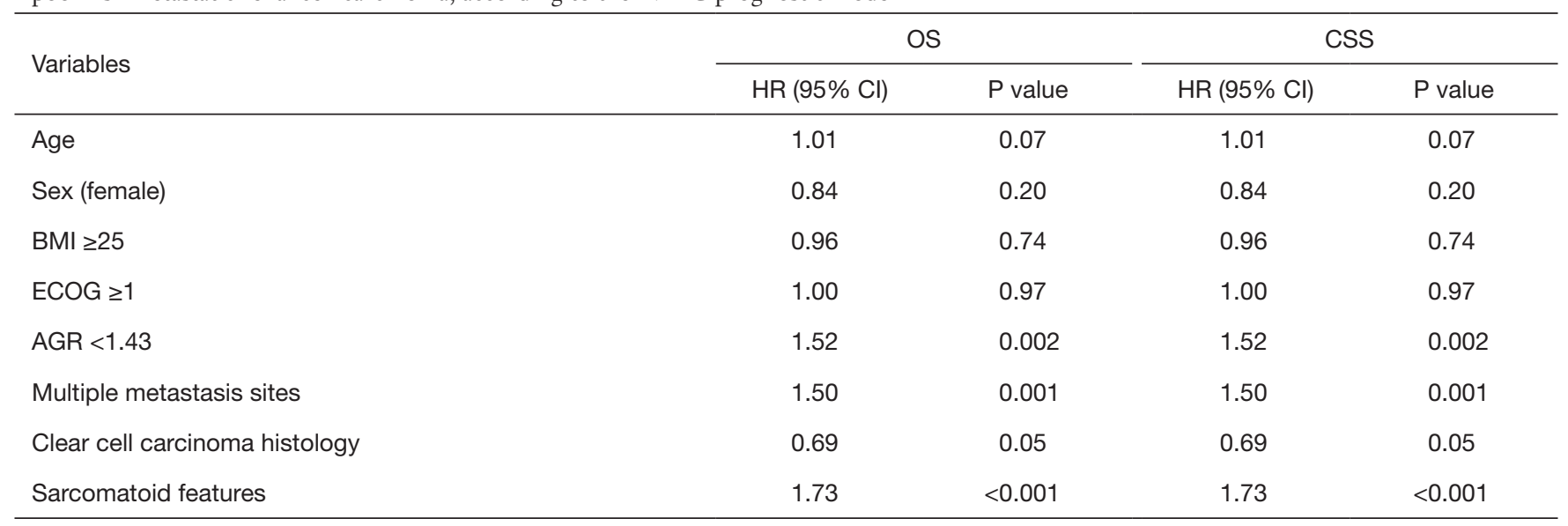

OS, overall survival; CSS, cancer-specific survival; IMDC, International Metastatic RCC Database Consortium Risk Model; BMI, body mass index; ECOG, Eastern Cooperative Oncology Group performance status; AGR, albumin globulin ratio.
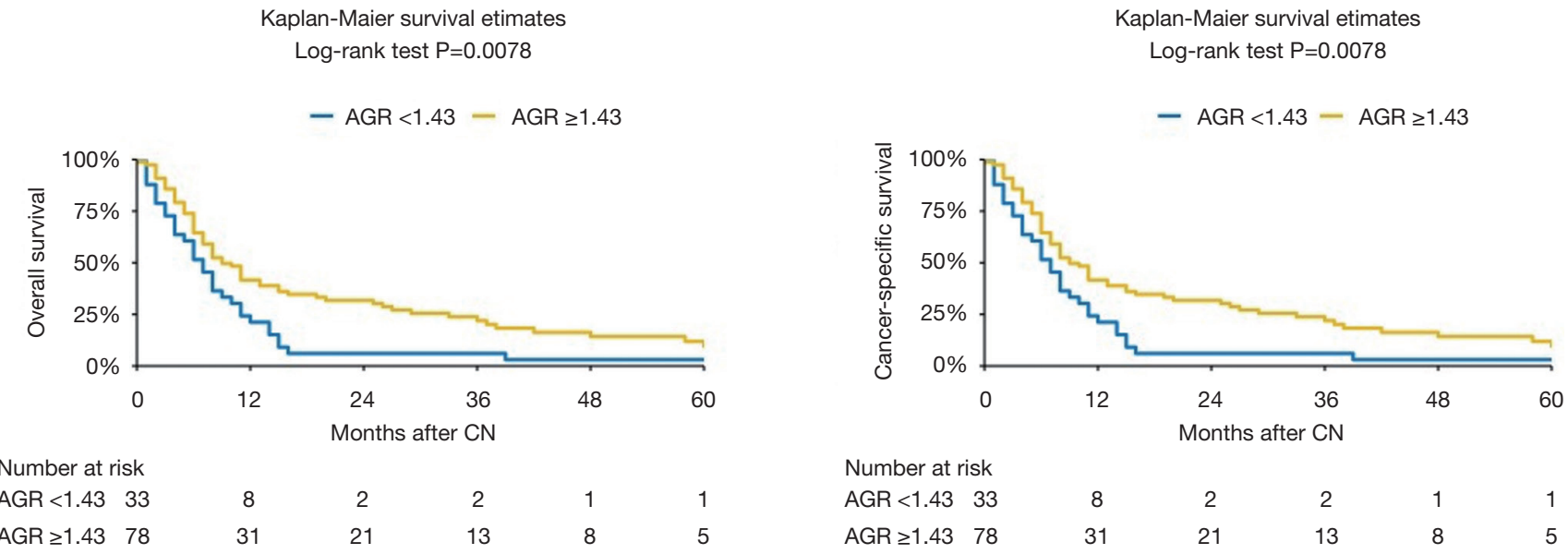

Figure 4 Overall and cancer-specific survival in patients treated with cytoreductive nephrectomy (CN) for metastatic renal cell carcinoma with sarcomatoid features, stratified according to preoperative serum albumin to globulin ratio (AGR) at a cut-off of 1.43 .

mRCC patients have not been tested and could be tested in the future studies.

\section{Conclusions}

Low AGR before CN was predicted to worse OS and CSS, particularly in patients with intermediate risk mRCC. It did, however, not add prognostically and clinically significant information beyond currently available clinicopathologic characteristics. This study should be the benchmark for future evaluation of AGR as a preoperative blood based prognostic biomarker in the era of checkpoint inhibitor.

\section{Acknowledgments}

Funding: None.

\section{Footnote}

Reporting Checklist: The authors have completed the STROBE reporting checklist. Available at http://dx.doi. org/10.21037/tau-20-1101 
Data Sharing Statement: Available at http://dx.doi. org/10.21037/tau-20-1101

Peer Review File: Available at http://dx.doi.org/10.21037/ tau-20-1101

Conflicts of Interest: All authors have completed the ICMJE uniform disclosure form (available at http://dx.doi. org/10.21037/tau-20-1101). Vitaly Margulis serves as an unpaid editorial board member of Translational Andrology and Urology from Nov 2018 to Oct 2020. The authors have no other conflicts of interest to declare.

Ethical Statement: The authors are accountable for all aspects of the work in ensuring that questions related to the accuracy or integrity of any part of the work are appropriately investigated and resolved. The study was conducted in accordance with the Declaration of Helsinki (as revised in 2013). This study was reviewed and approved by the University of Texas Southwestern (Dallas, Texas, USA) Institutional Review Board (UT SW IRB File 0698 26900). Because of the retrospective nature of the research, the requirement for informed consent was waived.

Open Access Statement: This is an Open Access article distributed in accordance with the Creative Commons Attribution-NonCommercial-NoDerivs 4.0 International License (CC BY-NC-ND 4.0), which permits the noncommercial replication and distribution of the article with the strict proviso that no changes or edits are made and the original work is properly cited (including links to both the formal publication through the relevant DOI and the license). See: https://creativecommons.org/licenses/by-nc-nd/4.0/.

\section{References}

1. Gupta K, Miller JD, Li JZ, et al. Epidemiologic and socioeconomic burden of metastatic renal cell carcinoma (mRCC): A literature review. Cancer Treat Rev 2008;34:193-205.

2. Heng DYC, Xie W, Regan MM, et al. Prognostic factors for overall survival in patients with metastatic renal cell carcinoma treated with vascular endothelial growth factortargeted agents: Results from a large, multicenter study. J Clin Oncol 2009;27:5794-9.

3. Ljungberg B, Bensalah K, Canfield S, et al. EAU guidelines on renal cell carcinoma. Arnhem, The Netherlands: EAU Guidelines Office, 2019.
4. Coussens LM, Werb Z. Inflammation and cancer. Nature 2002;420:860-7.

5. Park TJ, Cho YH, Chung HS, et al. Prognostic significance of platelet-lymphocyte ratio in patients receiving first-line tyrosine kinase inhibitors for metastatic renal cell cancer. Springerplus 2016;5:1889.

6. Gunduz S, Mutlu H, Tural D, et al. Platelet to lymphocyte ratio as a new prognostic for patients with metastatic renal cell cancer. Asia Pac J Clin Oncol 2015;11:288-92.

7. Lucca I, de Martino M, Hofbauer SL, et al. Comparison of the prognostic value of pretreatment measurements of systemic inflammatory response in patients undergoing curative resection of clear cell renal cell carcinoma. World J Urol 2015;33:2045-52.

8. Hutterer GC, Stoeckigt C, Stojakovic T, et al. Low preoperative lymphocyte-monocyte ratio (LMR) represents a potentially poor prognostic factor in nonmetastatic clear cell renal cell carcinoma. Urol Oncol 2014;32:1041-8.

9. Boissier R, Campagna J, Branger N, et al. The prognostic value of the neutrophil-lymphocyte ratio in renal oncology: A review. Urol Oncol 2017;35:135-41.

10. Gabay C, Kushner I. Acute-phase proteins and other systemic responses to inflammation. $\mathrm{N}$ Engl J Med 1999;340:448-54.

11. Kibrick AC, Clements AB. A comparative study of the serum albumin-globulin ratio, the cephalin-cholesterol flocculation, and the thymol turbidity tests for liver function. J Lab Clin Med 1948;33:662-71.

12. Chi J, Xie Q, Jia J, et al. Prognostic value of albumin/ globulin ratio in survival and lymph node metastasis in patients with cancer: A systematic review and metaanalysis. J Cancer 2018; 9:2341-48.

13. Lv GY, An L, Sun XD, et al. Pretreatment albumin to globulin ratio can serve as a prognostic marker in human cancers: a meta-analysis. Clin Chim Acta 2018;476:81-91.

14. Zhang $\mathrm{Y}$, Wang L, Lin S, et al. Preoperative albuminto-globulin ratio as a significant prognostic indicator in urologic cancers: A meta-analysis. Cancer Manag Res 2018;10:4695-708.

15. He J, Pan H, Liang W, et al. Prognostic effect of albuminto-globulin ratio in patients with solid tumors: A systematic review and meta-analysis. J Cancer 2017;8:4002-10.

16. Chen Z, Shao Y, Yao H, et al. Preoperative albumin to globulin ratio predicts survival in clear cell renal cell carcinoma patients. Oncotarget 2017;8:48291-302.

17. He X, Guo S, Chen D, et al. Preoperative Albumin to Globulin Ratio (AGR) as prognostic factor in renal cell carcinoma. J Cancer 2017;8:258-65. 
18. Koparal MY, Polat F, Çetin S, et al. Prognostic role of preoperative albumin to globulin ratio in predicting survival of clear cell renal cell carcinoma. Int Braz J Urol 2018;44:933-46.

19. Shariat SF, Lotan Y, Vickers A, et al. Statistical consideration for clinical biomarker research in bladder cancer. Urol Oncol 2010;28:389-400.

20. D'Andrea D, Soria F, Zehetmayer S, et al. Diagnostic accuracy, clinical utility and influence on decisionmaking of a methylation urine biomarker test in the surveillance of non-muscle-invasive bladder cancer. BJU Int 2019;123:959-67.

21. Pfensig C, Dominik A, Borufka L, et al. A New Application for Albumin Dialysis in Extracorporeal Organ Support: Characterization of a Putative Interaction Between Human Albumin and Proinflammatory Cytokines IL-6 and TNF $\alpha$. Artif Organs 2016;40:397-402.

22. Claesson-Welsh L. Vascular permeability - The essentials. Ups J Med Sci 2015;120:135-43.

23. Bozkaya Y, Erdem GU, Demirci NS, et al. Prognostic importance of the albumin to globulin ratio in metastatic

Cite this article as: Laukhtina E, Pradere B, D'Andrea D, Rosiello G, Luzzago S, Pecoraro A, Palumbo C, Knipper S, Karakiewicz PI, Margulis V, Quhal F, Sari Motlang R, Mostafaei H, Mori K, Schuettfort VM, Enikeev D, Shariat SF. Prognostic effect of preoperative serum albumin to globulin ratio in patients treated with cytoreductive nephrectomy for metastatic renal cell carcinoma. Transl Androl Urol 2021;10(2):609-619. doi: 10.21037/tau-20-1101 gastric cancer patients. Curr Med Res Opin 2019;35:275-82.

24. Shariat SF, Semjonow A, Lilja H, et al. Tumor markers in prostate cancer I: Blood-based markers. Acta Oncol 2011;50 Suppl 1:61-75.

25. Garje R, An J, Greco A, et al. The future of immunotherapy-based combination therapy in metastatic renal cell carcinoma. Cancers (Basel) 2020;12:143.

26. Nakanishi Y, Masuda T, Yamaguchi K, et al. Albuminglobulin ratio is a predictive biomarker of antitumor effect of anti-PD-1 antibody in patients with non-small cell lung cancer. Int J Clin Oncol 2020;25:74-81.

27. Fukuda H, Takagi T, Kondo T, et al. Predictive value of inflammation-based prognostic scores in patients with metastatic renal cell carcinoma treated with cytoreductive nephrectomy. Oncotarget 2018;9:14296-305.

28. Gu L, Ma X, Wang L, et al. Prognostic value of a systemic inflammatory response index in metastatic renal cell carcinoma and construction of a predictive model. Oncotarget 2016;8:52094-103.

29. Bensalah K, Montorsi F, Shariat SF. Challenges of Cancer Biomarker Profiling. Eur Urol 2007;52:1601-9. 